\title{
SHOOT DRY WEIGHT YIELD, BROMATOLOGICAL COMPOSITION, AND NUTRITIONAL STATUS OF ALFALFA CULTIVARS IN TROPICAL CONDITIONS
}

Adônis Moreira ${ }^{1}$, Reinaldo Paula Ferreira ${ }^{2}$, Duarte Vilela ${ }^{3}$, Larissa Alexandra Cardoso Moraes ${ }^{1}$, Alfredo Ribeiro de Freitas ${ }^{2}$

\footnotetext{
${ }^{1}$ Embrapa Soja, Londrina, Paraná State, Brazil

E-mail: amoreira@pq.cnpq.br; larissa.moraes@embrapa.br

${ }^{2}$ Embrapa Pecuária Sudeste, São Carlos, São Paulo State, Brazil

E-mail: reinaldo.ferreira@embrapa.br; alfribeiro@hotmail.com

${ }^{3}$ Embrapa Gado de Leite, Juiz de Fora, Minas Gerais State, Brazil

E-mail: duarte.vilela@embrapa.br
}

\section{ABSTRACT}

Improvement to animal genetics requires the supply of superior quality forage, such as alfalfa (Medicago sativa L.). Under tropical and subtropical conditions in Brazil, alfalfa cultivation has been restricted to the 'Crioula' cultivar, although studies have indicated that other cultivars have excellent potential of yield. The objective of this work was to evaluate the adaptation and longevity of 92 alfalfa cultivars to the tropical edaphoclimatic conditions by assessing shoot dry weight yield (SDWY), crude protein content (CP), neutral detergent fiber (NDF), acid detergent fiber (ADF), and nutrient content in SDWY. The 'Crioula', 'P 105', 'Rio Grande', and 'Springfield' cultivars were the most promising owing to their higher yield and lower reduction in SDWY. Crude protein (CP) content varied among cultivars, with the highest value found in 'Pro INTA Lujan' (24.5\%) and the lowest found in 'Pecos' (18.8\%). NDF content ranged from 25.8\% ('Key II') to $38.7 \%$ ('California 50 '), while ADF content ranged from $16.4 \%$ ('F 708') to $24.8 \%$ ('Diamond'). Nutrient levels of all cultivars were within the levels considered appropriate for tropical conditions.

Keywords: Medicago sativa, mineral nutrition, crude protein, neutral detergent fiber, acid detergent fiber

\section{PRODUÇÃO DE MATÉRIA SECA, COMPOSIÇÃO BROMATOLÓGICA E ESTADO NUTRICIONAL DE CULTIVARES DE ALFAFA EM CONDIÇÕES TROPICAIS}

\section{RESUMO}

A melhoria da genética animal exige o fornecimento de uma forragem de qualidade superior como a alfafa (Medicago sativa L.), que nas condições brasileira se restringe a cultivar 'Crioula'. O objetivo deste trabalho foi avaliar a adaptação e longevidade de 92 cultivares de alfafa para as 
condições edafoclimáticas dos trópicos quanto às produções de matéria seca da parte aérea (MSPA), teor de proteína bruta (PB), fibra em detergente neutro (FDN), fibra em detergente ácido (FDA) e teores de nutrientes na MSPA. As cultivares 'Crioula', 'P 105', 'Rio Grande' e 'Springfield' foram as mais promissoras por apresentarem maior produção e menor índice de redução na produção de MSPA. O teor de proteína bruta variou entre os cultivares, com valor maior para 'Pro INTA Lujan' (24,5\%) e menor para 'Pecos' $(18,8)$, enquanto para os teores de FDN e FDA variaram de 25,8\% ('Key II') a 38,7\% ('Califórnia 50') e de 16,4\% ('F 708') a 24,8\% ('Diamond'). Independentemente da cultivar, os teores dos nutrientes na matéria seca ficaram dentro dos níveis considerados adequados para o cultivo da alfafa.

Palavras-chave: Medicago sativa, nutrição mineral, proteína bruta, fibra detergente neutro, fibra detergente ácido

\section{INTRODUCTION}

Alfalfa (Medicago sativa L.) is a legume rich in minerals, protein, and vitamins A and C; it has broad phenotypic plasticity and can be grown in both tropical and temperate regions (MOREIRA et al., 1996). Despite this adaptation to different latitudes, photoperiod is one factor that may limit the introduction of new accessions, a limitation common in legumes. Photoperiod is considered a limiting factor in the choice of materials selected for programs at different latitudes worldwide (NUERNBERG et al., 1992; REZENDE et al., 2007).

Alfalfa is excellent quality forage, producing more protein than white clover (Trifolium repens L.) or corn (Zea mays L.) silage (RASSINI et al., 2007). The cultivar selection process, the photoperiod sensitivity is a characteristic that varies between cultivars and/or species; each cultivar/species may have a different critical photoperiod, above which flowering may or not occur. Other factors, such as temperature, soil moisture, nutritional status, weed presence, and planting density can influence grain or SDWY (SEDIYAMA, 1989; MOREIRA et al., 2007).

Forage species with morphological, physiological, and agronomic characteristics that maximize forage yield and quality are desirable (FERREIRA et al., 1999). To identify the alfalfa cultivars best adapted to a given environment, periodic evaluations by means of cuts are necessary to assess the relevant principal phenotypic characteristics (FREITAS et al., 2008). Thus, one can estimate both the genotypic variability of the available material and the repeatability of performance for each cultivar or progeny (FERREIRA et al., 2004). 
Interest in alfalfa crops has led to several studies evaluating cultivars, taking into consideration not only yield, but also adaptability and quality of forage (MOREIRA et al., 1996; BOTREL et al., 2002; FERREIRA et al., 2004; RASSINI et al., 2007). These studies aimed to obtain new cultivation options and/or genotypes that are more productive than the 'Crioula' cultivar. This cultivar is practically the only cultivar planted in Brazil, despite the excellent adaptability of alfalfa under different edaphoclimatic conditions.

The objective of this work was to evaluate the shoot fry weight yield (SDWY), bromatological qualities (acid detergent fiber $[\mathrm{ADF}]$, neutral detergent fiber [NDF], and crude protein [CP]), and nutritional status of 92 alfalfa accessions (cultivars), in addition to the 'Crioula' cultivar, for tropical conditions.

\section{MATERIAL AND METHODS}

A field trial was conducted at the Embrapa Pecuaria Sudeste located in São Carlos, São

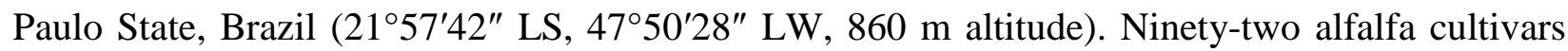
were planted in a Typic Oxisol with the following chemical attributes at the 0-20 cm depth: $\mathrm{pH}$ $\left(\mathrm{CaCl}_{2}\right)=5.2$, soil organic matter $(\mathrm{SOM})=17.0 \mathrm{~g} \mathrm{dm}^{-3}$, phosphorus $(\mathrm{P})$ available (ion exchange resin $)=13 \mathrm{mg} \mathrm{kg}^{-1}$, potassium $\left(\mathrm{K}^{+}\right)=2.3 \mathrm{cmol}_{\mathrm{c}} \mathrm{kg}^{-1}$, calcium $\left(\mathrm{Ca}^{2+}\right)=1.3 \mathrm{cmol}_{\mathrm{c}} \mathrm{kg}^{-1}$, magnesium $\left(\mathrm{Mg}^{2+}\right)=0.4 \mathrm{cmol}_{\mathrm{c}} \mathrm{kg}^{-1}$, aluminum $\left(\mathrm{Al}^{3+}\right)=0,3 \mathrm{cmol}_{\mathrm{c}} \mathrm{kg}^{-1}$, potential acidity $(\mathrm{H}+\mathrm{Al})=2.4 \mathrm{cmol}_{\mathrm{c}}$ $\mathrm{kg}^{-1}$, and cation exchange capacity $(\mathrm{CEC})=6.4 \mathrm{cmol}_{\mathrm{c}} \mathrm{kg}^{-1}$. The alfalfa cultivars were evaluated at three separate times, during three cuts.

Thirty days before alfalfa planting $5.0 \mathrm{Mg} \mathrm{ha}^{-1}$ of dolomite limestone ( $\mathrm{MgO}>13 \%$; CARVALHO et al., 1994) was applied to the site, and at sowing time $80 \mathrm{~kg} \mathrm{ha}^{-1}$ of $\mathrm{P}_{2} \mathrm{O}_{5}$ (simple superphosphate, $20 \%$ of $\mathrm{P}_{2} \mathrm{O}_{5}$ ), $100 \mathrm{~kg} \mathrm{ha}^{-1}$ of $\mathrm{K}_{2} \mathrm{O}$ (potassium chloride, $60 \% \mathrm{~K}_{2} \mathrm{O}$ ), and $30 \mathrm{~kg} \mathrm{ha}^{-}$ ${ }^{1}$ of fritted trace, FTE BR $12^{\circledR}$ (boron [B], 1.8\%; copper [Cu], 0.8\%; iron [Fe], 3.0\%; manganese [Mn], 2.0\%; molybdenum [Mo], 0.1\%; and zinc [Zn], 9.0\%) were applied. The seeds were inoculated with Shinorhizobium meliloti strains, and planting density was $20 \mathrm{~kg} \mathrm{ha}^{-1}$. After each cut, at the time of sowing basal fertilizer rate used was the potassium chloride $\left(\mathrm{KCl}, 60 \%\right.$ of $\left.\mathrm{K}_{2} \mathrm{O}\right)$ according to Moreira et al. (2008a). The alfalfa was irrigated using a central pivot, following an irrigation schedule according to Rassini (2007).

The experiment was set up as a randomized block design, with two replicates per cultivar, and repeated measures over time (FREITAS et al., 2011). The plot dimensions were $5.0 \mathrm{~m} \times 2.5$ 
$\mathrm{m}$, with a usable area of $3.6 \mathrm{~m}^{2}$. Five successive cuts based on the stage of plant development were made after two years of cultivation (14 previous cuts) in the usable area of each plot. Due to variation among cultivars, the time at which cuts were made varied with each cultivar. Cuts were made at $5.0 \mathrm{~cm}$ from the soil whenever each cultivar either had $10 \%$ of plants that were flowering or when the plants began to develop basal shoots. The characteristics of shoot dry weight yield (SDWY), crude protein (CP) content in SDWY, neutral detergent fiber (NDF), acid detergent fiber $(\mathrm{ADF})$ and total phosphorus $(\mathrm{P})$, potassium $(\mathrm{K})$, calcium $(\mathrm{Ca})$, magnesium $(\mathrm{Mg})$, sulfur $(\mathrm{S})$, boron (B), copper $(\mathrm{Cu})$, iron $(\mathrm{Fe})$, manganese $(\mathrm{Mn})$, and zinc $(\mathrm{Zn})$ were evaluated. Nutrient analyzes were performed according to the methodologies described by Malavolta et al. (1997), while the NDF and ADF analyzes were in accordance with AOAC (1995).

Results were analyzed as repeated measures (FREITAS et al. 2011) analysis of variance (ANOVA, $F$-tests), with means compared by Scott-Knott cluster test at $5 \%$ probability.

\section{RESULTS AND DISCUSSION}

There were significant differences between cultivars in relation to SDWY, CP, NDF, and ADF (Table 1). The 'Crioula', 'P 105', 'Rio Grande', and 'Springfield' cultivars had the highest cutting longevity in SDWY and produced, on average, more than $1000 \mathrm{~kg} \mathrm{ha}^{-1}$ per cut. Yields of all cultivars ranged from $1092.4 \mathrm{~kg} \mathrm{ha}^{-1}$ for the 'Crioula' cultivar to $287.3 \mathrm{~kg} \mathrm{ha}^{-1}$ for 'Eterna', and an average $728.7 \mathrm{~kg} \mathrm{ha}^{-1}$, indicating that the selection procedures based on which 'Crioula' was identified as a cultivar adapted to tropical and subtropical edaphoclimatic conditions successfully produced a high-yield, long-lasting cultivar in terms of SDWY.

Costa et al. (2006) quantified the SDWY of 28 alfalfa cultivars in Botucatu, São Paulo State, Brazil, and reported significant differences between cultivars, with a mean of $1620 \mathrm{~kg} \mathrm{ha}^{-1}$ of SDWY per cut, 55.1\% higher than that found in the present study (Table 1). This difference in productivity is mainly due to the evaluation period of the harvesting season since the data of the present work were obtained after two years of cultivation and successive cuts. Rassini et al. (2007) report the results of this initial two-year period. Verifying the longevity of alfalfa cuts is a major factor in the material selection process; according to Nuernberg et al. (1992) and Ferreira et al. (1999), well-managed alfalfa can be economically viable for up to four successive cutting years (MOREIRA et al., 1996). 
Table 1. Shoot dry weight yield (SDWY), crude protein (CP), neutral detergent fiber (NDF), and acid detergent fiber (ADF) content in alfalfa cultivars. São Carlos, São Paulo State, Brazil.

\begin{tabular}{|c|c|c|c|c|}
\hline Cultivars & $\begin{array}{l}\text { SDWY } \\
\mathrm{kg} \mathrm{ha}^{-1}\end{array}$ & $\begin{array}{c}\mathrm{CP} \\
\%\end{array}$ & $\begin{array}{c}\text { NDF } \\
\%\end{array}$ & $\begin{array}{c}\mathrm{ADF} \\
\%\end{array}$ \\
\hline 5683 & $934.8 \mathrm{c}$ & $22.7 \mathrm{~b}$ & $34.4 \mathrm{a}$ & $21.1 \mathrm{a}$ \\
\hline 5929 & $960.8 \mathrm{c}$ & $24.1 \mathrm{a}$ & $36.9 \mathrm{a}$ & $21.8 \mathrm{a}$ \\
\hline 5939 & $869.5 \mathrm{~d}$ & $20.7 \mathrm{c}$ & $37.3 \mathrm{a}$ & $22.4 \mathrm{a}$ \\
\hline 13 RR SUPREME & $629.6 \mathrm{i}$ & $19.8 \mathrm{~d}$ & $31.4 \mathrm{~b}$ & $22.9 \mathrm{a}$ \\
\hline $5683 \mathrm{~L}$ & $964.1 \mathrm{c}$ & $21.5 \mathrm{c}$ & $35.4 \mathrm{a}$ & $21.1 \mathrm{a}$ \\
\hline $58 \mathrm{~N} 58$ & $775.8 \mathrm{f}$ & $21.6 \mathrm{c}$ & $34.4 \mathrm{a}$ & $22.5 \mathrm{a}$ \\
\hline ACA 900 & $658.3 \mathrm{i}$ & $19.2 \mathrm{~d}$ & $38.5 \mathrm{a}$ & $21.6 \mathrm{a}$ \\
\hline ACA 901 & $626.1 \mathrm{i}$ & $19.2 \mathrm{~d}$ & $33.9 \mathrm{a}$ & $22.0 \mathrm{a}$ \\
\hline ACTIVA & $719.2 \mathrm{~g}$ & $22.2 \mathrm{c}$ & $33.5 \mathrm{a}$ & $20.8 \mathrm{a}$ \\
\hline ALFA 200 & $813.2 \mathrm{e}$ & $20.4 \mathrm{~d}$ & $33.4 \mathrm{a}$ & $22.1 \mathrm{a}$ \\
\hline AMERIGRAZE 701 & $809.8 \mathrm{e}$ & $20.1 \mathrm{~d}$ & $34.7 \mathrm{a}$ & $23.2 \mathrm{a}$ \\
\hline AURORA & $707.6 \mathrm{~h}$ & $22.6 b$ & $33.7 \mathrm{a}$ & $21.7 \mathrm{a}$ \\
\hline BACANA & $718.4 \mathrm{~g}$ & $19.3 \mathrm{~d}$ & $33.0 \mathrm{~b}$ & $23.9 \mathrm{a}$ \\
\hline BÁRBARA SP INTA & $816.0 \mathrm{e}$ & $22.7 \mathrm{~b}$ & $30.7 b$ & $18.1 \mathrm{~b}$ \\
\hline CALIFORNIA 50 & $820.2 \mathrm{e}$ & $21.3 \mathrm{c}$ & $38.7 \mathrm{a}$ & $21.9 \mathrm{a}$ \\
\hline CALIFÓRNIA 60 & $730.0 \mathrm{~g}$ & $21.7 \mathrm{c}$ & $32.4 \mathrm{~b}$ & $23.3 \mathrm{a}$ \\
\hline CANDOMBE & $744.4 \mathrm{~g}$ & $23.1 \mathrm{~b}$ & $32.6 b$ & $22.2 \mathrm{a}$ \\
\hline CORONADO & $787.7 \mathrm{f}$ & $21.5 \mathrm{c}$ & $32.1 \mathrm{~b}$ & $19.3 b$ \\
\hline COSTERA SP INTA & $560.2 \mathrm{k}$ & $23.4 \mathrm{~b}$ & $32.2 b$ & $17.7 \mathrm{~b}$ \\
\hline CRIOULA & $1092.4 \mathrm{a}$ & $22.7 \mathrm{~b}$ & $35.2 \mathrm{a}$ & $24.7 \mathrm{a}$ \\
\hline CUF 101 & $564.2 \mathrm{k}$ & $23.6 \mathrm{~b}$ & $35.2 \mathrm{a}$ & $22.3 \mathrm{a}$ \\
\hline DIAMOND & $945.2 \mathrm{c}$ & $21.9 \mathrm{c}$ & $38.5 \mathrm{a}$ & $24.8 \mathrm{a}$ \\
\hline DK 166 & $824.0 \mathrm{e}$ & $22.1 \mathrm{c}$ & $32.8 \mathrm{~b}$ & $21.5 \mathrm{a}$ \\
\hline DK 167 & $743.3 \mathrm{~g}$ & $21.0 \mathrm{c}$ & $35.8 \mathrm{a}$ & $23.6 \mathrm{a}$ \\
\hline DK 177 & $578.0 \mathrm{j}$ & $23.6 \mathrm{~b}$ & $33.4 \mathrm{a}$ & $20.2 b$ \\
\hline DK 181 & $974.8 \mathrm{c}$ & $20.8 \mathrm{c}$ & $35.7 \mathrm{a}$ & $21.2 \mathrm{a}$ \\
\hline DK 187 RR & $405.1 \mathrm{n}$ & $20.8 \mathrm{c}$ & $33.2 \mathrm{~b}$ & $20.7 \mathrm{a}$ \\
\hline DK 193 & $727.9 \mathrm{~g}$ & $24.8 \mathrm{a}$ & $36.4 \mathrm{a}$ & $17.4 \mathrm{~b}$ \\
\hline DK 194 & $709.7 \mathrm{~h}$ & $21.2 \mathrm{c}$ & $35.7 \mathrm{a}$ & $20.8 \mathrm{a}$ \\
\hline ESMERALDA SP INTA & $441.8 \mathrm{~m}$ & $23.6 \mathrm{~b}$ & $33.7 \mathrm{a}$ & $22.3 \mathrm{a}$ \\
\hline ETERNA & 287.30 & $24.6 \mathrm{a}$ & $32.7 \mathrm{~b}$ & $17.0 \mathrm{~b}$ \\
\hline EXPRESS & $860.4 \mathrm{e}$ & $22.3 b$ & $36.6 \mathrm{a}$ & $21.5 \mathrm{a}$ \\
\hline F 686 & $594.2 \mathrm{j}$ & $22.2 \mathrm{c}$ & $31.9 \mathrm{~b}$ & $20.8 \mathrm{a}$ \\
\hline F 708 & 507.51 & $23.4 \mathrm{~b}$ & $27.6 \mathrm{~b}$ & $16.5 b$ \\
\hline FLÓRIDA 77 & $817.1 \mathrm{e}$ & $22.7 \mathrm{~b}$ & $32.8 b$ & $23.8 \mathrm{a}$ \\
\hline GALA & $602.8 \mathrm{j}$ & $22.7 \mathrm{~b}$ & $31.5 b$ & $21.0 \mathrm{a}$ \\
\hline GAPP 969 & $652.4 \mathrm{i}$ & $21.6 \mathrm{c}$ & $31.2 \mathrm{~b}$ & $18.2 \mathrm{~b}$ \\
\hline GT 13 RR PLUS & 857.1 & $22.3 b$ & $29.1 b$ & $16.9 \mathrm{~b}$ \\
\hline HUNTERFIELD & $765.5 \mathrm{f}$ & $24.7 \mathrm{a}$ & $30.5 b$ & $23.5 \mathrm{a}$ \\
\hline KERN & $422.6 n$ & $25.2 \mathrm{a}$ & $26.8 b$ & $17.4 \mathrm{~b}$ \\
\hline KEY II & $630.4 \mathrm{i}$ & $24.2 \mathrm{a}$ & $25.8 \mathrm{~b}$ & $17.4 \mathrm{~b}$ \\
\hline LE SEMIT 711 & $549.0 \mathrm{k}$ & $22.8 \mathrm{~b}$ & $34.2 \mathrm{a}$ & $21.0 \mathrm{a}$ \\
\hline LE N 1 & $452.7 \mathrm{~m}$ & $20.9 \mathrm{c}$ & $36.7 \mathrm{a}$ & $22.1 \mathrm{a}$ \\
\hline LE N 2 & $936.6 \mathrm{c}$ & $20.8 \mathrm{c}$ & $35.7 \mathrm{a}$ & $21.7 \mathrm{a}$ \\
\hline LE N 3 & $924.5 \mathrm{~d}$ & $22.2 \mathrm{c}$ & $37.5 \mathrm{a}$ & $23.4 \mathrm{a}$ \\
\hline LE N 4 & $728.8 \mathrm{~g}$ & $20.6 c$ & $33.9 \mathrm{a}$ & $19.8 \mathrm{~b}$ \\
\hline LE SEMIT 711 & $898.9 \mathrm{~d}$ & $22.0 \mathrm{c}$ & $28.7 b$ & $19.1 \mathrm{~b}$ \\
\hline LUJAN & $730.2 \mathrm{~g}$ & $24.6 \mathrm{a}$ & $31.0 \mathrm{~b}$ & $19.8 b$ \\
\hline MARICOPA & $948.7 \mathrm{c}$ & $20.5 \mathrm{~d}$ & $34.2 \mathrm{a}$ & $23.5 \mathrm{a}$ \\
\hline
\end{tabular}

Continue... 
Table 1. Shoot dry weight yield (SDWY), crude protein (CP), neutral detergent fiber (NDF), and acid detergent fiber (ADF) content in alfalfa cultivars. São Carlos, São Paulo State, Brazil. (continue...)

\begin{tabular}{|c|c|c|c|c|}
\hline Cultivars & $\begin{array}{l}\text { SDWY } \\
\mathrm{kg} \mathrm{ha}^{-1}\end{array}$ & $\begin{array}{c}\mathrm{CP} \\
\% \\
\end{array}$ & $\begin{array}{c}\mathrm{NDF} \\
\% \\
\end{array}$ & $\begin{array}{c}\text { ADF } \\
\%\end{array}$ \\
\hline MAXIDOR & $603.7 j$ & $23.4 \mathrm{~b}$ & $36.2 \mathrm{a}$ & $22.1 \mathrm{a}$ \\
\hline MEDINA & $998.5 \mathrm{~b}$ & $22.0 \mathrm{c}$ & $31.2 \mathrm{~b}$ & $22.0 \mathrm{a}$ \\
\hline MONARCA & $664.2 \mathrm{i}$ & $22.9 \mathrm{~b}$ & $27.8 \mathrm{~b}$ & $16.5 b$ \\
\hline MONARCA SP INTA & 379.80 & $21.1 \mathrm{c}$ & $33.0 \mathrm{~b}$ & $18.1 \mathrm{~b}$ \\
\hline N 910 & $675.3 \mathrm{i}$ & $21.8 \mathrm{c}$ & $34.4 \mathrm{a}$ & $23.5 \mathrm{a}$ \\
\hline P 105 & $1073.3 \mathrm{a}$ & $24.3 \mathrm{a}$ & $30.1 \mathrm{~b}$ & $18.5 b$ \\
\hline P 30 & $581.3 \mathrm{j}$ & $22.9 \mathrm{c}$ & $32.4 \mathrm{~b}$ & $19.8 b$ \\
\hline P 5715 & $535.9 \mathrm{k}$ & $21.7 \mathrm{c}$ & $35.1 \mathrm{a}$ & $21.8 \mathrm{a}$ \\
\hline PATRICIA & $656.2 \mathrm{i}$ & $22.5 \mathrm{c}$ & $34.8 \mathrm{a}$ & $22.3 \mathrm{a}$ \\
\hline PECOS & $892.2 d$ & $18.6 \mathrm{~d}$ & $35.3 \mathrm{a}$ & $23.5 \mathrm{a}$ \\
\hline PERLA & $612.3 \mathrm{j}$ & $24.0 \mathrm{a}$ & $29.5 b$ & $19.2 \mathrm{~b}$ \\
\hline PERLA SP INTA & $662.1 \mathrm{i}$ & $21.2 \mathrm{c}$ & $34.5 \mathrm{a}$ & $20.7 \mathrm{a}$ \\
\hline PINTO & $924.9 \mathrm{~d}$ & $20.3 \mathrm{~d}$ & $37.0 \mathrm{a}$ & $22.7 \mathrm{a}$ \\
\hline PLATINO & $824.4 \mathrm{e}$ & $23.6 \mathrm{~b}$ & $41.1 \mathrm{a}$ & $26.3 \mathrm{a}$ \\
\hline PRIMAVERA 1 & $662.6 \mathrm{i}$ & $23.7 \mathrm{~b}$ & $33.3 \mathrm{a}$ & $20.4 b$ \\
\hline PRIMAVERA 2 & $822.8 \mathrm{e}$ & $23.4 \mathrm{~b}$ & $29.9 \mathrm{~b}$ & $17.6 \mathrm{~b}$ \\
\hline PRO INTA LUJAN & $552.8 \mathrm{k}$ & $24.5 \mathrm{a}$ & $28.7 \mathrm{~b}$ & $18.1 \mathrm{~b}$ \\
\hline PRO INTA PATRICIA & $661.4 \mathrm{i}$ & $22.7 \mathrm{c}$ & $31.1 \mathrm{~b}$ & $22.1 \mathrm{a}$ \\
\hline RIO GRANDE & $1032.6 b$ & $22.1 \mathrm{c}$ & $30.2 b$ & $19.8 b$ \\
\hline ROCIO & $772.3 f$ & $22.9 \mathrm{c}$ & $32.0 \mathrm{~b}$ & $17.7 \mathrm{~b}$ \\
\hline SEQUEL & $842.9 \mathrm{e}$ & $18.7 \mathrm{~d}$ & $35.4 \mathrm{a}$ & $20.3 b$ \\
\hline SEQUEL 2 & $868.1 \mathrm{e}$ & $23.3 \mathrm{c}$ & $36.9 \mathrm{a}$ & $21.3 \mathrm{a}$ \\
\hline SEQUEL HR & $670.1 \mathrm{i}$ & $22.2 \mathrm{c}$ & $33.4 \mathrm{a}$ & $20.5 b$ \\
\hline SIRIVER & $374.0 \mathrm{n}$ & $21.7 \mathrm{c}$ & $33.0 \mathrm{~b}$ & $19.0 \mathrm{~b}$ \\
\hline SIRIVER 2 & $905.1 \mathrm{~d}$ & $22.1 \mathrm{c}$ & $34.7 \mathrm{a}$ & $17.9 \mathrm{~b}$ \\
\hline SPRINGFIELD & $1004.5 b$ & $23.0 \mathrm{c}$ & $36.4 a$ & $19.1 \mathrm{~b}$ \\
\hline SPS 6550 & $751.3 \mathrm{~g}$ & $20.0 \mathrm{~d}$ & $35.0 \mathrm{a}$ & $22.5 \mathrm{a}$ \\
\hline SUNDOR & $951.0 \mathrm{c}$ & $21.0 \mathrm{c}$ & $36.1 \mathrm{a}$ & $18.9 \mathrm{~b}$ \\
\hline SUTTER & $599.9 \mathrm{j}$ & $23.9 \mathrm{a}$ & $33.8 \mathrm{a}$ & $22.3 \mathrm{a}$ \\
\hline TAHOE & $399.3 n$ & $23.0 \mathrm{c}$ & $31.6 b$ & $17.4 \mathrm{~b}$ \\
\hline TANGO & $393.5 n$ & $24.3 \mathrm{a}$ & $26.9 b$ & $16.9 \mathrm{~b}$ \\
\hline TOPPER & $985.6 \mathrm{~b}$ & $23.6 \mathrm{~b}$ & $33.5 \mathrm{a}$ & $21.0 \mathrm{a}$ \\
\hline TRINIDAD 87 & $928.0 \mathrm{~b}$ & $20.8 \mathrm{c}$ & $37.0 \mathrm{a}$ & $24.5 \mathrm{a}$ \\
\hline VICTORIA & $675.6 \mathrm{i}$ & $24.2 \mathrm{a}$ & $35.0 \mathrm{a}$ & $18.9 \mathrm{~b}$ \\
\hline VICTORIA SP INTA & $705.7 \mathrm{~h}$ & $21.3 \mathrm{c}$ & $36.1 \mathrm{a}$ & $22.2 \mathrm{a}$ \\
\hline WINTER & 509.91 & $22.0 \mathrm{c}$ & $30.7 \mathrm{~b}$ & $19.2 \mathrm{~b}$ \\
\hline WL 414 a & $657.9 \mathrm{i}$ & $22.4 \mathrm{c}$ & $33.3 \mathrm{a}$ & $19.9 \mathrm{~b}$ \\
\hline WL 414 b & $425.8 n$ & $23.7 \mathrm{~b}$ & $33.0 \mathrm{~b}$ & $19.1 \mathrm{~b}$ \\
\hline WL 442 & $787.3 \mathrm{f}$ & $21.5 \mathrm{c}$ & $31.1 \mathrm{~b}$ & $19.5 \mathrm{~b}$ \\
\hline Mean & 728.7 & 22.2 & 33.5 & 20.8 \\
\hline $\mathrm{CV}(\%)$ & 12.11 & 15.56 & 10.64 & 13.80 \\
\hline
\end{tabular}

*Means followed by distinct letters in the same column are statistically significant at the $5 \%$ probability level by Scott-Knott test. CV - coefficient of variation.

Crude protein content in SDWY ranged from $18.6 \%$ in 'Pecos' to $24.5 \%$ in 'Pro INTA Lujan', with an average of $22.2 \%$ among all cultivars (Table 1). However, when converted into protein content in SDWY $([\mathrm{CP} \times \mathrm{SDWY}] / 100)$, the highest values were observed in cultivar 'P 
105 ', with $259.9 \mathrm{~kg} \mathrm{ha}^{-1}$ of CP, and the lowest in 'Monarca SP INTA', with $79.9 \mathrm{~kg} \mathrm{ha}^{-1}$ of CP. Despite this variability, CP levels are well above those obtained in two previous studies. Moreira et al. (1996) studied nine alfalfa cultivars in four growing seasons and obtained an average of $17.7 \%$ of CP in SDWY. Moreira et al. (1997) studied sulfur (S) sources and rates and found an average $\mathrm{CP}$ content of $21.5 \%$. Differences in protein composition between cultivars can be attributed to the development cycle and differing environmental conditions, in which cultivars with lower $\mathrm{CP}$ content have higher growth rates (Costa et al. 2006). This corroborates the findings of Baligar et al. (2001) and Moreira et al. (2016), who reported that nutritional composition might vary between species and cultivars or varieties within the same plant species.

The NDF values, which ranged from $25.8 \%$ ('Key II') to $38.7 \%$ ('California 50 '), with an average content among cultivars of $33.5 \%$ (Table 1), were lower than the data obtained by Costa et al. (2006), who evaluated 28 alfalfa cultivars and obtained NDF values between $43.1 \%$ and $47.0 \%$ in the SDWY. The proportion of NDF of forage is important not only for the evaluation of its chemical composition, but also because the NDF is related to the maximum dry weight intake (COSTA et al., 2006); i.e., plants with higher NDF levels have lower consumption potential. According to Monteiro et al. (1998), there is a slight tendency for the most productive cultivars to exhibit lower NDF content, which can be inferred from our results, since, except for 'Crioula', the cultivars 'P 105', 'Rio Grande', and 'Springfield' had values below the average (33.5\%).

Acid detergent fiber (ADF) represents the least digestible portion of the forage cell wall (cellulose and lignin) (van SOEST et al., 1991). ADF content varied from 16.4\% ('F 708') to 24.8\% ('Diamond'), with an average of 20.8\%, and ADF content did not correlate with SDWY and CP content (Table 1). The ADF values found in alfalfa cultivars are well below those reported by Monteiro et al. (1998) and Costa et al. (2006), who obtained average levels of $31.7 \%$ and $32.4 \%$, respectively. In nutritional terms, Machado et al. (1998) and Monteiro et al. (1998) reported that the highest FDA content in SDWY is found in plants with the highest levels of cellulose and lignin, due to the higher conversion rates of photosynthetic products into structural tissues.

The relative magnitude of $\mathrm{P}, \mathrm{K}, \mathrm{Ca}, \mathrm{Mg}$, and $\mathrm{S}$ content in the SDWY was as follows: $\mathrm{K}$ > $\mathrm{Ca}>\mathrm{P}>\mathrm{Mg}>\mathrm{S}$. The content of these macronutrients varied between cultivars from 2.9 ('Sequel') to $3.9 \mathrm{~g} \mathrm{P} \mathrm{kg}^{-1}$ ('Siriver'); 31.5 ('Siriver') to $39.8 \mathrm{~g} \mathrm{~K} \mathrm{~kg}^{-1}$ ('Maxidor'); 6.7 ('Barbara SP INTA') to $11.2 \mathrm{~g} \mathrm{Ca} \mathrm{kg}^{-1}$ (5939); 2.4 ('Florida 77') to $3.3 \mathrm{~g} \mathrm{Mg} \mathrm{kg}^{-1}$ ('F 686'); and 1.7 ('P5715') to $2.9 \mathrm{~g} \mathrm{~S} \mathrm{~kg}^{-1}$ ('Le Semit 711'), respectively (Table 2). These are like those obtained by Moreira et al. (2008a) 
and Moreira et al. (2008b) within the ranges indicated by them as adequate levels for alfalfa cultivation in tropical conditions. The relative magnitude of micronutrient content was as follows: $\mathrm{Fe}>\mathrm{Mn}>\mathrm{Zn}>\mathrm{B}>\mathrm{Cu}$ (Table 3), with content ranging from 15.8 ('Monarch') to $33.4 \mathrm{mg} \mathrm{B} \mathrm{kg}^{-1}$ ('Springfield'); 11.7 ('Siriver 2') to $22.2 \mathrm{mg} \mathrm{Cu} \mathrm{kg}^{-1}$ ('Springfield'); 229.2 ('DK 193') to $608.5 \mathrm{mg}$ Fe kg-1 ('Barbara SP INTA'); 24.8 ('WL 414 b') to 36.9 mg Mn kg-1 ('Patricia'); and 20.2 ('5939') to $35.0 \mathrm{mg} \mathrm{Zn} \mathrm{kg}^{-1}$ ('Siriver'), respectively. Despite of the yield, these are, independently of cultivar, within the appropriate levels for the cultivation and use of alfalfa in the tropics (MOREIRA et al., 2000; MOREIRA et al., 2008a).

Table 2. Macronutrients (P, K, Ca, Mg, and S) content of shoot dry weight in alfalfa cultivars. São Carlos, São Paulo State, Brazil.

\begin{tabular}{|c|c|c|c|c|c|}
\hline Cultivars & $\begin{array}{c}\mathrm{P} \\
\mathrm{g} \mathrm{kg}^{-1}\end{array}$ & $\begin{array}{c}\mathrm{K} \\
\mathrm{g} \mathrm{kg}^{-1}\end{array}$ & $\begin{array}{c}\mathrm{Ca} \\
\mathrm{g} \mathrm{kg}^{-1}\end{array}$ & $\begin{array}{c}\mathrm{Mg} \\
\mathrm{g} \mathrm{kg}^{-1}\end{array}$ & $\begin{array}{c}\mathrm{S} \\
\mathrm{g} \mathrm{kg}^{-1}\end{array}$ \\
\hline 5683 & $3.3 \mathrm{~b}$ & $36.7 \mathrm{a}$ & $10.0 \mathrm{a}$ & $2.7 \mathrm{a}$ & $2.5 \mathrm{a}$ \\
\hline 5929 & $3.6 \mathrm{a}$ & $35.3 \mathrm{a}$ & $10.2 \mathrm{a}$ & $3.0 \mathrm{a}$ & $2.2 \mathrm{a}$ \\
\hline 5939 & $3.3 b$ & $31.8 \mathrm{~b}$ & $11.2 \mathrm{a}$ & $3.2 \mathrm{a}$ & $2.1 \mathrm{a}$ \\
\hline 13 RR SUPREME & $3.1 \mathrm{~b}$ & $33.8 \mathrm{~b}$ & $9.4 \mathrm{a}$ & $3.1 \mathrm{a}$ & $2.2 \mathrm{a}$ \\
\hline $5683 \mathrm{~L}$ & $3.4 \mathrm{a}$ & $32.2 \mathrm{~b}$ & $8.1 \mathrm{~b}$ & $2.7 \mathrm{a}$ & $2.1 \mathrm{a}$ \\
\hline $58 \mathrm{~N} 58$ & $3.3 \mathrm{~b}$ & $34.7 \mathrm{a}$ & $9.1 \mathrm{a}$ & $3.0 \mathrm{a}$ & $2.4 \mathrm{a}$ \\
\hline ACA 900 & $3.4 \mathrm{a}$ & $34.3 \mathrm{a}$ & $8.6 \mathrm{a}$ & $2.9 \mathrm{a}$ & $2.1 \mathrm{a}$ \\
\hline ACA 901 & $3.4 \mathrm{a}$ & $33.1 \mathrm{~b}$ & $7.1 \mathrm{~b}$ & $2.7 \mathrm{a}$ & $2.2 \mathrm{a}$ \\
\hline ACTIVA & $3.3 b$ & $35.2 \mathrm{a}$ & $8.6 \mathrm{a}$ & $2.9 \mathrm{a}$ & $2.3 \mathrm{a}$ \\
\hline ALFA 200 & $3.3 b$ & $34.4 \mathrm{a}$ & $7.5 \mathrm{~b}$ & $3.0 \mathrm{a}$ & $2.3 \mathrm{a}$ \\
\hline AMERIGRAZE 701 & $3.0 \mathrm{~b}$ & $38.1 \mathrm{a}$ & $9.6 \mathrm{a}$ & $3.1 \mathrm{a}$ & $2.1 \mathrm{a}$ \\
\hline AURORA & $3.3 \mathrm{~b}$ & $33.8 \mathrm{~b}$ & $10.0 \mathrm{a}$ & $2.8 \mathrm{a}$ & $2.1 \mathrm{a}$ \\
\hline BACANA & $3.3 b$ & $34.1 \mathrm{a}$ & $7.1 \mathrm{~b}$ & $2.8 \mathrm{a}$ & $2.2 \mathrm{a}$ \\
\hline BÁRBARA SP INTA & $3.7 \mathrm{a}$ & $36.8 \mathrm{a}$ & $6.7 \mathrm{~b}$ & $2.9 \mathrm{a}$ & $2.4 \mathrm{a}$ \\
\hline CALIFORNIA 50 & $3.2 \mathrm{~b}$ & $33.9 \mathrm{~b}$ & $9.3 \mathrm{a}$ & $3.2 \mathrm{a}$ & $1.9 \mathrm{a}$ \\
\hline CALIFÓRNIA 60 & $3.2 \mathrm{~b}$ & $34.3 \mathrm{a}$ & $9.4 \mathrm{a}$ & $2.8 \mathrm{a}$ & $2.1 \mathrm{a}$ \\
\hline CANDOMBE & $3.3 b$ & $38.7 \mathrm{a}$ & $9.6 \mathrm{a}$ & $2.9 \mathrm{a}$ & $2.6 \mathrm{a}$ \\
\hline CORONADO & $3.3 \mathrm{~b}$ & $36.4 \mathrm{a}$ & $8.6 \mathrm{a}$ & $3.0 \mathrm{a}$ & $2.2 \mathrm{a}$ \\
\hline COSTERA SP INTA & $3.4 \mathrm{a}$ & $33.8 \mathrm{~b}$ & $8.5 \mathrm{a}$ & $3.4 \mathrm{a}$ & $2.3 \mathrm{a}$ \\
\hline CRIOULA & $3.1 \mathrm{~b}$ & $34.5 \mathrm{a}$ & 10.3 & $3.1 \mathrm{a}$ & $2.1 \mathrm{a}$ \\
\hline CUF 101 & $3.8 \mathrm{a}$ & $35.9 \mathrm{a}$ & $8.7 \mathrm{a}$ & $3.0 \mathrm{a}$ & $2.3 \mathrm{a}$ \\
\hline DIAMOND & $3.1 \mathrm{~b}$ & $36.9 \mathrm{a}$ & $8.8 \mathrm{a}$ & $2.9 \mathrm{a}$ & $2.4 \mathrm{a}$ \\
\hline DK 166 & $3.0 \mathrm{~b}$ & $36.0 \mathrm{a}$ & $9.3 \mathrm{a}$ & $2.7 \mathrm{a}$ & $2.0 \mathrm{a}$ \\
\hline DK 167 & $2.9 \mathrm{~b}$ & $32.9 \mathrm{~b}$ & $7.9 \mathrm{~b}$ & $2.9 \mathrm{a}$ & $2.2 \mathrm{a}$ \\
\hline DK 177 & $3.6 \mathrm{a}$ & $37.2 \mathrm{a}$ & $10.5 \mathrm{a}$ & $2.9 \mathrm{a}$ & $2.3 \mathrm{a}$ \\
\hline DK 181 & $3.0 \mathrm{~b}$ & $35.1 \mathrm{a}$ & $10.5 \mathrm{a}$ & $2.9 \mathrm{a}$ & $2.4 \mathrm{a}$ \\
\hline DK 187 RR & $3.3 b$ & $38.6 \mathrm{a}$ & $8.1 \mathrm{~b}$ & $3.1 \mathrm{a}$ & $2.2 \mathrm{a}$ \\
\hline DK 193 & $3.4 \mathrm{a}$ & $35.2 \mathrm{a}$ & $9.7 \mathrm{a}$ & $3.2 \mathrm{a}$ & $2.4 \mathrm{a}$ \\
\hline DK 194 & $3.3 b$ & $35.9 \mathrm{a}$ & $8.3 \mathrm{a}$ & $2.8 \mathrm{a}$ & $2.0 \mathrm{a}$ \\
\hline ESMERALDA SP INTA & $3.2 b$ & $34.3 \mathrm{a}$ & $9.6 \mathrm{a}$ & $3.2 \mathrm{a}$ & $2.4 \mathrm{a}$ \\
\hline ETERNA & $3.8 \mathrm{a}$ & $37.9 \mathrm{a}$ & $7.5 b$ & $3.2 \mathrm{a}$ & $2.2 \mathrm{a}$ \\
\hline EXPRESS & $3.0 \mathrm{~b}$ & $37.3 \mathrm{a}$ & $10.3 \mathrm{a}$ & $2.7 \mathrm{a}$ & $2.5 \mathrm{a}$ \\
\hline F 686 & $3.3 b$ & $32.9 \mathrm{~b}$ & $10.5 \mathrm{a}$ & $3.3 \mathrm{a}$ & $2.3 \mathrm{a}$ \\
\hline F 708 & $3.4 \mathrm{a}$ & $31.7 \mathrm{~b}$ & $8.5 \mathrm{a}$ & $2.9 \mathrm{a}$ & $2.3 \mathrm{a}$ \\
\hline FLÓRIDA 77 & $3.2 b$ & $33.2 \mathrm{~b}$ & $8.3 \mathrm{a}$ & $2.4 \mathrm{a}$ & $2.3 \mathrm{a}$ \\
\hline
\end{tabular}

Continue... 
Table 2. Macronutrients (P, K, Ca, Mg, and S) content of shoot dry weight in alfalfa cultivars. São Carlos, São Paulo State, Brazil (continue...).

\begin{tabular}{|c|c|c|c|c|c|}
\hline Cultivars & $\begin{array}{c}\mathrm{P} \\
\mathrm{g} \mathrm{kg}^{-1}\end{array}$ & $\begin{array}{c}\mathrm{K} \\
\mathrm{g} \mathrm{kg}^{-1}\end{array}$ & $\begin{array}{c}\mathrm{Ca} \\
\mathrm{g} \mathrm{kg}^{-1}\end{array}$ & $\begin{array}{c}\mathrm{Mg} \\
\mathrm{g} \mathrm{kg}^{-1}\end{array}$ & $\begin{array}{c}\mathrm{S} \\
\mathrm{g} \mathrm{kg}^{-1}\end{array}$ \\
\hline GALA & $3.5 \mathrm{a}$ & $38.9 \mathrm{a}$ & $8.6 \mathrm{a}$ & $3.2 \mathrm{a}$ & $2.7 \mathrm{a}$ \\
\hline GAPP 969 & $3.2 b$ & $33.2 \mathrm{~b}$ & $9.3 \mathrm{a}$ & $2.9 \mathrm{a}$ & $2.3 \mathrm{a}$ \\
\hline GT 13 RR PLUS & $3.4 \mathrm{a}$ & $34.4 \mathrm{a}$ & $7.9 \mathrm{~b}$ & $3.0 \mathrm{a}$ & $2.4 \mathrm{a}$ \\
\hline HUNTERFIELD & $3.4 \mathrm{a}$ & $34.9 \mathrm{a}$ & $8.3 \mathrm{a}$ & $2.8 \mathrm{a}$ & $2.8 \mathrm{a}$ \\
\hline KERN & $3.6 \mathrm{a}$ & $33.4 \mathrm{~b}$ & $6.9 \mathrm{~b}$ & $2.8 \mathrm{a}$ & $2.4 \mathrm{a}$ \\
\hline KEY II & $3.8 \mathrm{a}$ & $33.9 \mathrm{~b}$ & $6.8 b$ & $3.0 \mathrm{a}$ & $2.4 \mathrm{a}$ \\
\hline LE SEMIT 711 & $3.8 \mathrm{a}$ & $38.0 \mathrm{a}$ & $8.2 \mathrm{a}$ & $2.7 \mathrm{a}$ & $2.9 \mathrm{a}$ \\
\hline LE N 1 & $3.5 \mathrm{a}$ & $36.1 \mathrm{a}$ & $8.3 \mathrm{a}$ & $2.7 \mathrm{a}$ & $2.4 \mathrm{a}$ \\
\hline LE N 2 & $3.2 \mathrm{~b}$ & $35.9 \mathrm{a}$ & $7.8 \mathrm{~b}$ & $2.7 \mathrm{a}$ & $2.6 \mathrm{a}$ \\
\hline LE N 3 & $3.3 b$ & $37.7 \mathrm{a}$ & $10.4 \mathrm{a}$ & $3.2 \mathrm{a}$ & $2.5 \mathrm{a}$ \\
\hline LE N 4 & $3.0 \mathrm{~b}$ & $31.9 \mathrm{~b}$ & $7.3 b$ & $2.7 \mathrm{a}$ & $2.1 \mathrm{a}$ \\
\hline LE SEMIT 711 & $3.6 \mathrm{a}$ & $35.3 \mathrm{a}$ & $9.2 \mathrm{a}$ & $3.1 \mathrm{a}$ & $2.3 \mathrm{a}$ \\
\hline LUJAN & $3.4 \mathrm{a}$ & $37.5 \mathrm{a}$ & $7.7 \mathrm{~b}$ & $3.0 \mathrm{a}$ & $2.3 \mathrm{a}$ \\
\hline MARICOPA & $3.0 \mathrm{~b}$ & $33.2 \mathrm{~b}$ & $10.2 \mathrm{a}$ & $3.2 \mathrm{a}$ & $2.3 \mathrm{a}$ \\
\hline MAXIDOR & $3.2 \mathrm{~b}$ & $39.8 \mathrm{a}$ & $8.5 \mathrm{a}$ & $2.5 \mathrm{a}$ & $2.4 \mathrm{a}$ \\
\hline MEDINA & $3.3 \mathrm{~b}$ & $34.5 \mathrm{a}$ & $9.0 \mathrm{a}$ & $2.9 \mathrm{a}$ & $2.3 \mathrm{a}$ \\
\hline MONARCA & $3.5 \mathrm{a}$ & $37.8 \mathrm{a}$ & $8.1 \mathrm{~b}$ & $3.0 \mathrm{a}$ & $2.5 \mathrm{a}$ \\
\hline MONARCA SP INTA & $3.5 \mathrm{a}$ & $34.7 \mathrm{a}$ & $7.7 \mathrm{~b}$ & $3.2 \mathrm{a}$ & $2.3 \mathrm{a}$ \\
\hline N 910 & $3.2 b$ & $34.4 \mathrm{a}$ & $8.1 \mathrm{~b}$ & $3.0 \mathrm{a}$ & $2.0 \mathrm{a}$ \\
\hline P 105 & $3.4 \mathrm{a}$ & $33.8 \mathrm{~b}$ & $9.3 \mathrm{a}$ & $3.0 \mathrm{a}$ & $2.5 \mathrm{a}$ \\
\hline P 30 & $3.9 \mathrm{a}$ & $37.5 \mathrm{a}$ & $7.4 \mathrm{~b}$ & $3.0 \mathrm{a}$ & $2.0 \mathrm{a}$ \\
\hline P 5715 & $3.4 \mathrm{a}$ & $35.5 \mathrm{a}$ & $7.4 \mathrm{~b}$ & $3.0 \mathrm{a}$ & $1.8 \mathrm{a}$ \\
\hline PATRICIA & $3.6 \mathrm{a}$ & $32.0 \mathrm{~b}$ & $8.5 \mathrm{a}$ & $3.0 \mathrm{a}$ & $2.3 \mathrm{a}$ \\
\hline PECOS & $3.1 \mathrm{~b}$ & $35.3 \mathrm{a}$ & $9.1 \mathrm{a}$ & $3.0 \mathrm{a}$ & $2.4 \mathrm{a}$ \\
\hline PERLA & $3.4 \mathrm{a}$ & $34.4 \mathrm{a}$ & $6.8 b$ & $2.8 \mathrm{a}$ & $2.4 \mathrm{a}$ \\
\hline PERLA SP INTA & $3.1 \mathrm{~b}$ & $33.7 \mathrm{~b}$ & $10.2 \mathrm{a}$ & $3.1 \mathrm{a}$ & $2.7 \mathrm{a}$ \\
\hline PINTO & $3.1 \mathrm{~b}$ & $36.0 \mathrm{a}$ & $7.4 \mathrm{~b}$ & $3.0 \mathrm{a}$ & $2.0 \mathrm{a}$ \\
\hline PLATINO & $3.3 b$ & $35.8 \mathrm{a}$ & $8.0 \mathrm{~b}$ & $2.8 \mathrm{a}$ & $2.3 \mathrm{a}$ \\
\hline PRIMAVERA 1 & $3.6 \mathrm{a}$ & $35.7 \mathrm{a}$ & $9.6 \mathrm{a}$ & $2.8 \mathrm{a}$ & $2.5 \mathrm{a}$ \\
\hline PRIMAVERA 2 & $3.5 \mathrm{a}$ & $35.0 \mathrm{a}$ & $9.6 \mathrm{a}$ & $3.3 \mathrm{a}$ & $2.1 \mathrm{a}$ \\
\hline PRO INTA LUJAN & $3.1 \mathrm{~b}$ & $36.0 \mathrm{a}$ & $8.8 \mathrm{a}$ & $2.9 \mathrm{a}$ & $2.4 \mathrm{a}$ \\
\hline PRO INTA PATRICIA & $3.4 \mathrm{a}$ & $37.2 \mathrm{a}$ & $8.4 \mathrm{a}$ & $2.7 \mathrm{a}$ & $2.4 \mathrm{a}$ \\
\hline RIO GRANDE & $3.3 b$ & $33.2 \mathrm{~b}$ & $7.3 b$ & $3.0 \mathrm{a}$ & $2.4 \mathrm{a}$ \\
\hline ROCIO & $3.5 \mathrm{a}$ & $35.4 \mathrm{a}$ & $7.3 \mathrm{~b}$ & $3.1 \mathrm{a}$ & $2.5 \mathrm{a}$ \\
\hline SEQUEL & $3.2 \mathrm{~b}$ & $36.2 \mathrm{a}$ & $8.5 \mathrm{a}$ & $3.3 \mathrm{a}$ & $1.8 \mathrm{a}$ \\
\hline SEQUEL 2 & $3.1 \mathrm{~b}$ & $33.9 \mathrm{~b}$ & $9.2 \mathrm{a}$ & $3.1 \mathrm{a}$ & $2.6 \mathrm{a}$ \\
\hline SEQUEL HR & $3.3 \mathrm{~b}$ & $35.7 \mathrm{a}$ & $8.4 \mathrm{a}$ & $2.9 \mathrm{a}$ & $2.2 \mathrm{a}$ \\
\hline SIRIVER & $3.9 \mathrm{a}$ & $31.5 \mathrm{~b}$ & $7.3 b$ & $3.0 \mathrm{a}$ & $2.4 \mathrm{a}$ \\
\hline SIRIVER 2 & $2.9 \mathrm{~b}$ & $35.3 \mathrm{a}$ & $10.2 \mathrm{a}$ & $2.6 \mathrm{a}$ & $2.3 \mathrm{a}$ \\
\hline SPRINGFIELD & $3.6 \mathrm{a}$ & $35.5 \mathrm{a}$ & $9.9 \mathrm{a}$ & $2.9 \mathrm{a}$ & $2.4 \mathrm{a}$ \\
\hline SPS 6550 & $3.0 \mathrm{~b}$ & $35.5 \mathrm{a}$ & $8.5 \mathrm{a}$ & $2.6 \mathrm{a}$ & $2.4 \mathrm{a}$ \\
\hline SUNDOR & $3.2 \mathrm{~b}$ & $32.0 \mathrm{~b}$ & $8.8 \mathrm{a}$ & $2.8 \mathrm{a}$ & $2.2 \mathrm{a}$ \\
\hline SUTTER & $3.4 \mathrm{a}$ & $36.9 \mathrm{a}$ & $7.7 \mathrm{~b}$ & $2.6 \mathrm{a}$ & $2.5 \mathrm{a}$ \\
\hline TAHOE & $3.4 \mathrm{a}$ & $34.1 \mathrm{a}$ & $8.8 \mathrm{a}$ & $2.7 \mathrm{a}$ & $2.5 \mathrm{a}$ \\
\hline TANGO & $3.7 \mathrm{a}$ & $35.1 \mathrm{a}$ & $7.7 \mathrm{~b}$ & $3.0 \mathrm{a}$ & $2.3 \mathrm{a}$ \\
\hline TOPPER & $3.7 \mathrm{a}$ & $35.9 \mathrm{a}$ & $8.4 \mathrm{a}$ & $2.9 \mathrm{a}$ & $2.6 \mathrm{a}$ \\
\hline TRINIDAD 87 & $3.1 \mathrm{~b}$ & $37.2 \mathrm{a}$ & $9.3 \mathrm{a}$ & $3.1 \mathrm{a}$ & $2.2 \mathrm{a}$ \\
\hline VICTORIA & $3.4 \mathrm{a}$ & $33.4 \mathrm{~b}$ & $7.7 \mathrm{~b}$ & $2.9 \mathrm{a}$ & $2.5 \mathrm{a}$ \\
\hline VICTORIA SP INTA & $3.2 \mathrm{~b}$ & $37.0 \mathrm{a}$ & $8.5 \mathrm{a}$ & $3.1 \mathrm{a}$ & $2.0 \mathrm{a}$ \\
\hline WINTER & $3.5 \mathrm{a}$ & $35.3 \mathrm{a}$ & $8.3 \mathrm{a}$ & $2.9 \mathrm{a}$ & $2.8 \mathrm{a}$ \\
\hline
\end{tabular}


Table 2. Macronutrients (P, K, Ca, Mg, and S) content of shoot dry weight in alfalfa cultivars. São Carlos, São Paulo State, Brazil (continue...).

\begin{tabular}{cccccc}
\hline Cultivars & $\begin{array}{c}\mathrm{P} \\
\mathrm{g} \mathrm{kg}^{-1}\end{array}$ & $\begin{array}{c}\mathrm{K} \\
\mathrm{g} \mathrm{kg}^{-1}\end{array}$ & $\begin{array}{c}\mathrm{Ca} \\
\mathrm{g} \mathrm{kg}^{-1}\end{array}$ & $\begin{array}{c}\mathrm{Mg} \\
\mathrm{g} \mathrm{kg}^{-1}\end{array}$ & $\begin{array}{c}\mathrm{S} \\
\mathrm{g} \mathrm{kg}^{-1}\end{array}$ \\
\hline WL 414 a & $3.3 \mathrm{~b}$ & $32.9 \mathrm{~b}$ & $8.3 \mathrm{a}$ & $2.8 \mathrm{a}$ & $2.3 \mathrm{a}$ \\
WL 414 b & $3.4 \mathrm{a}$ & $38.1 \mathrm{a}$ & $8.9 \mathrm{a}$ & $2.7 \mathrm{a}$ & $2.6 \mathrm{a}$ \\
WL 442 & $3.4 \mathrm{a}$ & $36.5 \mathrm{a}$ & $7.3 \mathrm{~b}$ & $2.9 \mathrm{a}$ & $2.1 \mathrm{a}$ \\
\hline Mean & 3.2 & 36.9 & 8.0 & 2.8 & 2.2 \\
CV $(\%)$ & 12.44 & 13.51 & 12.44 & 11.93 & 16.17 \\
\hline
\end{tabular}

*Means followed by distinct letters in the same column are statistically significant at the 5\% probability level by ScottKnott test. CV - coefficient of variation.

Table 3. Micronutrients (B, $\mathrm{Cu}, \mathrm{Fe}, \mathrm{Mn}$, and $\mathrm{Zn}$ ) content of shoot dry weight in alfalfa cultivars. São Carlos, São Paulo State, Brazil.

\begin{tabular}{|c|c|c|c|c|c|}
\hline Cultivars & $\begin{array}{c}\mathrm{B} \\
\mathrm{mg} \mathrm{kg}^{-1}\end{array}$ & $\begin{array}{c}\mathrm{Cu} \\
\mathrm{mg} \mathrm{kg}^{-1}\end{array}$ & $\begin{array}{c}\mathrm{Fe} \\
\mathrm{mg} \mathrm{kg}^{-1}\end{array}$ & $\begin{array}{c}\mathrm{Mn} \\
\mathrm{mg} \mathrm{kg}^{-1}\end{array}$ & $\begin{array}{c}\mathrm{Zn} \\
\mathrm{mg} \mathrm{kg}^{-1}\end{array}$ \\
\hline 5683 & $21.2 \mathrm{c}$ & $12.5 \mathrm{~b}$ & $320.3 a$ & $28.2 \mathrm{a}$ & $25.9 \mathrm{~b}$ \\
\hline 5929 & $27.1 \mathrm{~b}$ & $19.0 \mathrm{a}$ & $368.2 \mathrm{a}$ & $31.0 \mathrm{a}$ & $24.8 b$ \\
\hline 5939 & $27.3 b$ & $19.0 \mathrm{a}$ & $299.4 b$ & $29.4 \mathrm{a}$ & $20.2 b$ \\
\hline 13 RR SUPREME & $20.4 \mathrm{c}$ & $12.0 \mathrm{~b}$ & $331.3 \mathrm{a}$ & $35.7 \mathrm{a}$ & $26.5 b$ \\
\hline $5683 \mathrm{~L}$ & $28.3 \mathrm{a}$ & $20.5 \mathrm{a}$ & $458.4 \mathrm{a}$ & $27.1 \mathrm{a}$ & $27.5 \mathrm{a}$ \\
\hline $58 \mathrm{~N} 58$ & $22.3 c$ & $19.8 \mathrm{a}$ & $302.3 \mathrm{a}$ & $26.6 \mathrm{a}$ & $23.0 \mathrm{~b}$ \\
\hline ACA 900 & $22.6 c$ & $16.7 \mathrm{~b}$ & $280.1 \mathrm{~b}$ & $26.3 \mathrm{a}$ & $23.3 b$ \\
\hline ACA 901 & $32.6 \mathrm{a}$ & $17.1 \mathrm{a}$ & $373.7 \mathrm{a}$ & $30.8 \mathrm{a}$ & $29.9 \mathrm{a}$ \\
\hline ACTIVA & $23.9 \mathrm{c}$ & $16.6 \mathrm{~b}$ & $359.3 \mathrm{a}$ & $30.3 a$ & $26.7 \mathrm{a}$ \\
\hline ALFA 200 & $24.7 \mathrm{~b}$ & $17.5 \mathrm{a}$ & $472.1 \mathrm{a}$ & $30.4 \mathrm{a}$ & $30.2 \mathrm{a}$ \\
\hline AMERIGRAZE 701 & $22.2 \mathrm{c}$ & $13.0 \mathrm{~b}$ & $327.2 \mathrm{a}$ & $31.2 \mathrm{a}$ & $22.6 \mathrm{~b}$ \\
\hline AURORA & $26.2 b$ & $21.5 \mathrm{a}$ & $298.5 b$ & $24.3 \mathrm{a}$ & $22.3 b$ \\
\hline BACANA & $29.3 \mathrm{a}$ & $18.9 \mathrm{a}$ & $386.5 \mathrm{a}$ & $30.2 \mathrm{a}$ & $28.8 \mathrm{a}$ \\
\hline BÁRBARA SP INTA & $20.5 c$ & $18.1 \mathrm{a}$ & $608.5 \mathrm{a}$ & $37.2 \mathrm{a}$ & $31.3 \mathrm{a}$ \\
\hline CALIFORNIA 50 & $26.9 b$ & $17.5 \mathrm{a}$ & $276.5 \mathrm{a}$ & $26.7 \mathrm{a}$ & $22.1 \mathrm{~b}$ \\
\hline CALIFÓRNIA 60 & $24.5 b$ & $12.8 \mathrm{~b}$ & $318.1 \mathrm{a}$ & $25.3 \mathrm{a}$ & $25.1 \mathrm{~b}$ \\
\hline CANDOMBE & $26.0 \mathrm{~b}$ & $18.9 \mathrm{a}$ & $313.2 \mathrm{a}$ & $26.6 \mathrm{a}$ & $22.7 b$ \\
\hline CORONADO & $22.9 \mathrm{c}$ & $17.7 \mathrm{a}$ & $314.9 \mathrm{a}$ & $33.6 \mathrm{a}$ & $28.8 \mathrm{a}$ \\
\hline COSTERA SP INTA & $21.6 \mathrm{c}$ & $18.8 \mathrm{a}$ & $436.6 \mathrm{a}$ & $28.8 \mathrm{a}$ & $29.0 \mathrm{a}$ \\
\hline CRIOULA & $18.4 \mathrm{c}$ & $16.1 \mathrm{~b}$ & $346.9 \mathrm{a}$ & $29.0 \mathrm{a}$ & $27.8 \mathrm{a}$ \\
\hline CUF 101 & $22.3 c$ & $18.9 \mathrm{a}$ & $344.2 \mathrm{a}$ & $32.0 \mathrm{a}$ & $29.2 \mathrm{a}$ \\
\hline DIAMOND & $24.5 \mathrm{~b}$ & $19.9 \mathrm{a}$ & $311.9 \mathrm{a}$ & $26.7 \mathrm{a}$ & $24.7 \mathrm{~b}$ \\
\hline DK 166 & $24.4 \mathrm{~b}$ & $20.5 \mathrm{a}$ & $316.5 \mathrm{a}$ & $24.6 \mathrm{a}$ & $21.1 \mathrm{~b}$ \\
\hline DK 167 & $28.6 \mathrm{a}$ & $12.7 \mathrm{~b}$ & $352.8 \mathrm{a}$ & $28.7 \mathrm{a}$ & $26.3 b$ \\
\hline DK 177 & $19.0 \mathrm{c}$ & $12.5 b$ & $299.8 b$ & $29.8 \mathrm{a}$ & $27.6 \mathrm{a}$ \\
\hline DK 181 & $17.1 \mathrm{c}$ & $12.8 \mathrm{~b}$ & $268.9 \mathrm{~b}$ & $29.6 \mathrm{a}$ & $23.1 \mathrm{~b}$ \\
\hline DK 187 RR & $23.5 \mathrm{c}$ & $16.9 \mathrm{~b}$ & $325.9 \mathrm{a}$ & $30.4 \mathrm{a}$ & $25.4 \mathrm{~b}$ \\
\hline DK 193 & $16.7 \mathrm{c}$ & $19.5 \mathrm{a}$ & $229.2 \mathrm{~b}$ & $30.6 \mathrm{a}$ & $24.6 \mathrm{~b}$ \\
\hline DK 194 & $25.8 b$ & $17.3 \mathrm{a}$ & $297.1 b$ & $30.5 a$ & $26.2 b$ \\
\hline ESMERALDA SP INTA & $21.5 \mathrm{c}$ & $14.9 \mathrm{~b}$ & $324.6 \mathrm{a}$ & $28.6 \mathrm{a}$ & $27.2 \mathrm{a}$ \\
\hline ETERNA & $19.2 \mathrm{c}$ & $20.3 \mathrm{a}$ & $407.7 \mathrm{a}$ & $26.4 \mathrm{a}$ & $29.8 \mathrm{a}$ \\
\hline EXPRESS & $18.9 \mathrm{c}$ & $13.0 \mathrm{~b}$ & $345.4 \mathrm{a}$ & $29.7 \mathrm{a}$ & $22.8 \mathrm{~b}$ \\
\hline F 686 & $21.1 \mathrm{c}$ & $16.3 b$ & $376.0 \mathrm{a}$ & $31.3 \mathrm{a}$ & $25.5 b$ \\
\hline F 708 & $18.3 \mathrm{c}$ & $15.3 b$ & $324.5 \mathrm{a}$ & $30.4 \mathrm{a}$ & $28.8 \mathrm{a}$ \\
\hline FLÓRIDA 77 & $24.1 \mathrm{c}$ & $13.8 \mathrm{~b}$ & $289.0 \mathrm{~b}$ & $30.7 \mathrm{a}$ & $28.5 \mathrm{a}$ \\
\hline GALA & $21.4 \mathrm{c}$ & $17.2 \mathrm{a}$ & $427.0 \mathrm{a}$ & $33.3 \mathrm{a}$ & $28.8 \mathrm{a}$ \\
\hline GAPP 969 & $33.2 \mathrm{~b}$ & $19.2 \mathrm{a}$ & $370.0 \mathrm{a}$ & $34.7 \mathrm{a}$ & $29.1 \mathrm{a}$ \\
\hline
\end{tabular}


Table 3. Micronutrients (B, $\mathrm{Cu}, \mathrm{Fe}, \mathrm{Mn}$, and $\mathrm{Zn}$ ) content of shoot dry weight in alfalfa cultivars. São Carlos, São Paulo State, Brazil (continue...).

\begin{tabular}{|c|c|c|c|c|c|}
\hline Cultivars & $\begin{array}{c}\mathrm{B} \\
\mathrm{mg} \mathrm{kg}^{-1}\end{array}$ & $\begin{array}{c}\mathrm{Cu} \\
\mathrm{mg} \mathrm{kg}^{-1}\end{array}$ & $\begin{array}{c}\mathrm{Fe} \\
\mathrm{mg} \mathrm{kg}^{-1}\end{array}$ & $\begin{array}{c}\mathrm{Mn} \\
\mathrm{mg} \mathrm{kg}^{-1}\end{array}$ & $\begin{array}{c}\mathrm{Zn} \\
\mathrm{mg} \mathrm{kg}^{-1}\end{array}$ \\
\hline GT 13 RR PLUS & $27.3 b$ & $19.2 \mathrm{a}$ & $356.9 \mathrm{a}$ & $32.5 \mathrm{a}$ & $28.6 \mathrm{a}$ \\
\hline HUNTERFIELD & $21.5 \mathrm{c}$ & $13.6 \mathrm{~b}$ & $395.8 \mathrm{a}$ & $26.2 \mathrm{a}$ & $27.0 \mathrm{a}$ \\
\hline KERN & $18.7 \mathrm{c}$ & $16.7 \mathrm{~b}$ & $409.2 \mathrm{a}$ & $28.5 \mathrm{a}$ & $28.2 \mathrm{a}$ \\
\hline KEY II & $24.0 \mathrm{c}$ & $16.5 \mathrm{~b}$ & $564.6 \mathrm{a}$ & $30.2 \mathrm{a}$ & $31.3 \mathrm{a}$ \\
\hline LE SEMIT 711 & $23.2 \mathrm{c}$ & $17.4 \mathrm{a}$ & $309.3 \mathrm{a}$ & $32.6 \mathrm{a}$ & $28.6 \mathrm{a}$ \\
\hline LE N 1 & $22.0 \mathrm{c}$ & $14.7 \mathrm{~b}$ & $349.5 \mathrm{a}$ & $31.2 \mathrm{a}$ & $26.8 \mathrm{a}$ \\
\hline LE N 2 & $22.9 \mathrm{c}$ & $14.9 \mathrm{~b}$ & $270.5 b$ & $27.5 \mathrm{a}$ & $24.7 \mathrm{~b}$ \\
\hline LE N 3 & $24.3 b$ & $16.9 \mathrm{~b}$ & $331.1 \mathrm{a}$ & $32.9 \mathrm{a}$ & $23.7 \mathrm{~b}$ \\
\hline LE N 4 & $30.0 \mathrm{~b}$ & $14.8 \mathrm{~b}$ & $284.1 \mathrm{~b}$ & $29.3 \mathrm{a}$ & $23.5 \mathrm{~b}$ \\
\hline LE SEMIT 711 & $21.6 \mathrm{c}$ & $18.4 \mathrm{a}$ & $511.0 \mathrm{a}$ & $31.7 \mathrm{a}$ & $23.5 b$ \\
\hline LUJAN & $20.7 \mathrm{c}$ & $16.5 \mathrm{~b}$ & $400.6 a$ & $29.5 \mathrm{a}$ & $27.2 \mathrm{a}$ \\
\hline MARICOPA & $22.7 \mathrm{c}$ & $14.0 \mathrm{~b}$ & $308.6 a$ & $27.4 \mathrm{a}$ & $23.2 \mathrm{~b}$ \\
\hline MAXIDOR & $20.8 \mathrm{c}$ & $14.6 \mathrm{~b}$ & $414.2 \mathrm{a}$ & $27.7 \mathrm{a}$ & $25.6 \mathrm{~b}$ \\
\hline MEDINA & $22.0 \mathrm{c}$ & $19.8 \mathrm{a}$ & $301.1 \mathrm{a}$ & $25.8 \mathrm{a}$ & $23.4 \mathrm{~b}$ \\
\hline MONARCA & $15.8 \mathrm{c}$ & $16.7 \mathrm{~b}$ & $379.3 \mathrm{a}$ & $34.2 \mathrm{a}$ & $30.7 \mathrm{a}$ \\
\hline MONARCA SP INTA & $26.1 \mathrm{~b}$ & $14.0 \mathrm{~b}$ & $374.6 \mathrm{a}$ & $30.7 \mathrm{a}$ & $26.0 \mathrm{~b}$ \\
\hline N 910 & $19.5 \mathrm{c}$ & $18.6 \mathrm{a}$ & $344.8 \mathrm{a}$ & $26.4 \mathrm{a}$ & $24.3 \mathrm{a}$ \\
\hline P 105 & $23.7 \mathrm{c}$ & $13.2 \mathrm{~b}$ & $372.0 \mathrm{a}$ & $28.4 \mathrm{a}$ & $26.2 b$ \\
\hline P 30 & $28.7 \mathrm{a}$ & $18.1 \mathrm{a}$ & $387.9 \mathrm{a}$ & $31.4 \mathrm{a}$ & $29.8 \mathrm{a}$ \\
\hline P 5715 & $25.2 \mathrm{~b}$ & $18.5 \mathrm{a}$ & $327.7 \mathrm{a}$ & $30.9 \mathrm{a}$ & $27.7 \mathrm{a}$ \\
\hline PATRICIA & $25.8 \mathrm{~b}$ & $15.0 \mathrm{~b}$ & $466.6 \mathrm{a}$ & $36.9 \mathrm{a}$ & $33.3 \mathrm{a}$ \\
\hline PECOS & $22.7 \mathrm{c}$ & $11.9 \mathrm{~b}$ & $294.5 b$ & $29.8 \mathrm{a}$ & $25.0 \mathrm{~b}$ \\
\hline PERLA & $23.2 \mathrm{c}$ & $16.9 \mathrm{~b}$ & $388.0 \mathrm{a}$ & $26.9 \mathrm{a}$ & $27.6 \mathrm{a}$ \\
\hline PERLA SP INTA & $20.4 \mathrm{c}$ & $14.8 \mathrm{~b}$ & $346.4 \mathrm{a}$ & $30.8 \mathrm{a}$ & $26.9 \mathrm{a}$ \\
\hline PINTO & $24.5 b$ & $19.4 \mathrm{a}$ & $370.0 \mathrm{a}$ & $31.9 \mathrm{a}$ & $26.7 \mathrm{a}$ \\
\hline PLATINO & $30.5 \mathrm{a}$ & $14.8 \mathrm{~b}$ & $635.7 \mathrm{a}$ & $34.3 \mathrm{a}$ & $30.7 \mathrm{a}$ \\
\hline PRIMAVERA 1 & $23.5 \mathrm{c}$ & $13.6 \mathrm{~b}$ & $433.9 \mathrm{a}$ & $32.8 \mathrm{a}$ & $26.3 b$ \\
\hline PRIMAVERA 2 & $31.4 \mathrm{a}$ & $18.4 \mathrm{a}$ & $405.7 \mathrm{a}$ & $29.1 \mathrm{a}$ & $21.9 \mathrm{~b}$ \\
\hline PROINTA LUJAN & $20.3 c$ & $15.4 \mathrm{~b}$ & $328.6 \mathrm{a}$ & $30.5 \mathrm{a}$ & $28.9 \mathrm{a}$ \\
\hline PROINTA PATRICIA & $22.7 \mathrm{c}$ & $13.5 \mathrm{~b}$ & $319.3 \mathrm{a}$ & $27.1 \mathrm{a}$ & $28.0 \mathrm{a}$ \\
\hline RIO GRANDE & $21.0 \mathrm{c}$ & $17.6 \mathrm{a}$ & $424.3 \mathrm{a}$ & $33.4 \mathrm{a}$ & $29.3 \mathrm{a}$ \\
\hline ROCIO & $31.2 \mathrm{a}$ & $18.0 \mathrm{a}$ & $315.2 \mathrm{a}$ & $27.5 \mathrm{a}$ & $28.5 \mathrm{a}$ \\
\hline SEQUEL & $25.7 \mathrm{~b}$ & $19.2 \mathrm{a}$ & $358.4 \mathrm{a}$ & $30.8 \mathrm{a}$ & $26.9 \mathrm{a}$ \\
\hline SEQUEL 2 & $27.1 \mathrm{~b}$ & $19.5 \mathrm{a}$ & $282.4 \mathrm{~b}$ & $27.1 \mathrm{a}$ & $22.9 \mathrm{~b}$ \\
\hline SEQUEL HR & $26.6 \mathrm{~b}$ & $18.6 \mathrm{a}$ & $371.2 \mathrm{a}$ & $30.6 a$ & $26.3 b$ \\
\hline SIRIVER & $27.1 \mathrm{~b}$ & $21.8 \mathrm{a}$ & $633.7 \mathrm{a}$ & $40.1 \mathrm{a}$ & $35.0 \mathrm{a}$ \\
\hline SIRIVER 2 & $22.5 \mathrm{c}$ & $11.7 \mathrm{~b}$ & $337.1 \mathrm{a}$ & $36.7 \mathrm{a}$ & $28.1 \mathrm{a}$ \\
\hline SPRINGFIELD & $33.4 \mathrm{a}$ & $22.2 \mathrm{a}$ & $309.0 \mathrm{a}$ & $30.7 \mathrm{a}$ & $25.9 \mathrm{~b}$ \\
\hline SPS 6550 & $21.8 \mathrm{c}$ & $14.8 \mathrm{~b}$ & $290.0 \mathrm{~b}$ & $37.5 \mathrm{a}$ & $25.0 \mathrm{~b}$ \\
\hline SUNDOR & $24.7 \mathrm{~b}$ & $21.6 \mathrm{a}$ & $301.9 \mathrm{a}$ & $23.6 \mathrm{a}$ & $23.0 \mathrm{~b}$ \\
\hline SUTTER & $26.4 \mathrm{~b}$ & $13.0 \mathrm{~b}$ & $271.1 \mathrm{~b}$ & $25.2 \mathrm{a}$ & $26.9 \mathrm{a}$ \\
\hline TAHOE & $22.7 \mathrm{c}$ & $15.2 b$ & $383.8 \mathrm{a}$ & $36.2 \mathrm{a}$ & $31.0 \mathrm{a}$ \\
\hline TANGO & $20.3 c$ & $14.0 \mathrm{~b}$ & $369.9 \mathrm{a}$ & $31.5 \mathrm{a}$ & $28.8 \mathrm{a}$ \\
\hline TOPPER & $23.3 \mathrm{c}$ & $18.5 \mathrm{a}$ & $349.7 \mathrm{a}$ & $29.0 \mathrm{a}$ & $25.5 \mathrm{~b}$ \\
\hline TRINIDAD 87 & $22.4 \mathrm{c}$ & $15.4 \mathrm{~b}$ & $336.9 \mathrm{a}$ & $35.0 \mathrm{a}$ & $24.3 b$ \\
\hline VICTORIA & $25.4 \mathrm{~b}$ & $14.3 \mathrm{~b}$ & $352.4 \mathrm{a}$ & $26.1 \mathrm{a}$ & $26.2 \mathrm{~b}$ \\
\hline VICTORIA SP INTA & $24.0 \mathrm{c}$ & $17.0 \mathrm{a}$ & $290.5 b$ & $31.7 \mathrm{a}$ & $22.5 b$ \\
\hline WINTER & $26.2 b$ & $14.4 \mathrm{~b}$ & $421.7 \mathrm{a}$ & $39.3 a$ & $30.0 \mathrm{a}$ \\
\hline
\end{tabular}

Continue... 
Table 3. Micronutrients (B, $\mathrm{Cu}, \mathrm{Fe}, \mathrm{Mn}$, and $\mathrm{Zn}$ ) content of shoot dry weight in alfalfa cultivars. São Carlos, São Paulo State, Brazil (continue...).

\begin{tabular}{cccccc}
\hline Cultivars & $\begin{array}{c}\mathrm{B} \\
\mathrm{mg} \mathrm{kg}\end{array}$ & $\begin{array}{c}\mathrm{Cu} \\
\mathrm{mg} \mathrm{kg}^{-1}\end{array}$ & $\begin{array}{c}\mathrm{Fe} \\
\mathrm{mg} \mathrm{kg}^{-1}\end{array}$ & $\begin{array}{c}\mathrm{Mn} \\
\mathrm{mg} \mathrm{kg}^{-1}\end{array}$ & $\begin{array}{c}\mathrm{Zn} \\
\mathrm{m} \mathrm{kg}^{-1}\end{array}$ \\
\hline WL 414 a & $23.9 \mathrm{c}$ & $18.5 \mathrm{a}$ & $328.4 \mathrm{a}$ & $33.5 \mathrm{a}$ & $28.8 \mathrm{a}$ \\
WL 414 b & $18.9 \mathrm{c}$ & $13.5 \mathrm{~b}$ & $300.2 \mathrm{a}$ & $24.8 \mathrm{a}$ & $27.4 \mathrm{a}$ \\
WL 442 & $26.2 \mathrm{~b}$ & $14.6 \mathrm{~b}$ & $331.9 \mathrm{a}$ & $32.4 \mathrm{a}$ & $26.5 \mathrm{~b}$ \\
\hline Mean & 23.3 & 15.1 & 376.3 & 40.9 & 32.8 \\
CV $(\%)$ & 17.07 & 15.98 & 19.21 & 17.58 & 14.43 \\
\hline
\end{tabular}

*Means followed by distinct letters in the same column are statistically significant at the $5 \%$ probability level by Scott-Knott test. CV - coefficient of variation.

\section{CONCLUSIONS}

The 'Crioula', 'P 105', 'Rio Grande', and 'Springfield' alfalfa cultivars had high cutting longevity and SDWY.

The cultivars 'Eterna', 'Monarch INTA', 'Siriver', 'Tahoe', and 'Tango' had yields of less than $400 \mathrm{~kg} \mathrm{ha}^{-1}$ per cut and were considered the least promising because of the lower SDWY.

In alfalfa cultivars, the bromatological composition (CP, ADF, and NDF) and nutritional status (macro and micronutrients) in SDWY were within the levels considered adequate in the literature for alfalfa cultivation in the tropics.

\section{ACKNOWLEDGEMENTS}

To the laboratory of plant mineral nutrition and bromatology of Embrapa Pecuária Sudeste (CPPSE) for analyzes, and to National Council for Research and Development (CNPq) scholarship for the first author.

\section{REFERENCES}

AOAC - Association OF Official Analytical Chemists. 1995. Official Methods of Analysis. Washington, D.C.: AOAC.

BALIGAR, V.C.; FAGERIA, N.K.; HE. Z.L. 2001. Nutrient use efficiency in plants. Communications in Soil Science and Plant Analysis, Athens, v. 32, n. 7-8, p. 921-950.

BALSALOBRE, M.A.A.; CORSI, M; SANTOS, P.M.; VIEIRA, I.; CÁRDENAS, R.R. 2003. Nutritional quality of irrigated Tanzania grass under three post-grazed stubbles intensities. Revista Brasileira de Zootecnia, Viçosa, v. 32, n. 3, p. 519-528.

BOTREL, M.A.; EVANGELISTA, A.R.; VIANA, M.C.M.; ALVIM, M.J.; XAVIER. D.F. 2002. Avaliação de cultivares de alfafa em diferentes regiões do Estado de Minas Gerais. Juiz de Fora: Embrapa Gado de Leite. 8 p.

CARVALHO, J.G.; ASSIS, R.P.; MOREIRA, A. 1994. Necessidade de calagem para cultura da alfafa. In: BOTREL, M.A.; ALVIM, M.J.; PASSOS, L.P.; VILELA, D. (Org.). Workshop 
sobre potencial forrageiro da alfafa (Medicago sativa L.) nos trópicos. Juiz de Fora: EMBRAPA-CNPGL, v. 1, p. 117-126.

COSTA, C.; MEIRELLES, P.R.L.; VIEIRA. M.E.Q. 2006. Dry matter yield and chemical composition of twenty-eight alfalfa (Medicago sativa L.) cultivars grown in Botucatu-SP. Veterinária e Zootecnia, Botucatu, v. 12, n. 1, p. 42-51.

FERREIRA, R.P.; BOTREL, M.A.; PEREIRA, A.V.; CRUZ, C.D. 1999. Evaluation of alfalfa cultivars and estimates of repeatability coefficient of forage traits. Pesquisa Agropecuária Brasileira, Brasília, v. 34, n. 6, p. 995-1002.

FERREIRA, R.P.; BOTREL, M.A.; RUGGIERI, A.C.; PEREIRA, A.V.; COELHO, A.D.F. LEDO, F.J.S.; CRUZ, C.D. 2004. Adaptability and stability of alfalfa cultivars in relation to different yielding dates. Ciência Rural, Santa Maria, v. 34, n. 1, p. 265-269, 2004.

FREITAS, A.R.; BARIONI JÚNIOR, W., FERREIRA, R.P., CRUZ, C.D.; MOREIRA, A.; VILELA, VILELA, D. 2008. Exploratory data analysis techniques in cultivars of alfalfa. Revista Brasileira de Zootecnia, Viçosa, v. 37, n. 9, p. 1531-1536.

FREITAS, A.R.; FERREIRA, R.P.; MOREIRA. A. 2011. Data analysis of repeated measures by means of general linear model and mixed model. Revista de Ciências Agrárias, Belém, v. 54, n. 3, p. 214-224.

MACHADO, A.O.; CECATO, U.; MIRA, R.T.; PEREIRA, L.A.F.; DAMASCENO, J.C. 1998. Evaluations of chemical composition and in vitro dry matter digestibility of cultivars and accesses of Panicum maximum Jacq. under two cutting heights. Revista Brasileira de Zootecnia, Viçosa, v. 27, n. 5, p. 1057-1063.

MONTEIRO, A.L.G.; COSTA, C.; SILVEIRA. A.C. 1998. Dry matter production and seasonal distribution and chemical composition of alfalfa cultivates (Medicago sativa L.). Revista Brasileira de Zootecnia, Viçosa, v. 27, n .4, p. 868-874.

MOREIRA, A.; BERNARDI, A.C.C.; RASSINI J.B. 2008a. Correção do solo, estado nutricional e adubação da alfafa. In: FERREIRA, R.P.; RASSINI, J.B.; RODRIGUES, A.A.; FREITAS, A.R.; CAMARGO, A.C.; MENDONÇA, F.C. (Eds.). Cultivo e utilização da alfafa nos trópicos, São Carlos: Embrapa Pecuária Sudeste. p. 95-137.

MOREIRA, A.; HEINRICHS, R.; FREITAS A.R. 2008b. Phosphorus and magnesium ratio on soil fertility, nutritional status, and yield of alfalfa. Revista Brasileira de Zootecnia, Viçosa, v. 37. n. 6, p. 984-989.

MOREIRA, A.; BERNARDI, A.C.C.; RASSINI, J.B.; FERREIRA, R.P.; OLIVEIRA, P.PA. 2007. Fertilidade do solo e nutrição mineral da alfafa cultivada nos trópicos. São Carlos: Embrapa Pecuária Sudeste. 40 p.

MOREIRA, A.; CARVALHO, J.G.; EVANGELISTA. A.R. 1997. Levels of sulfur effects on alfalfa production and mineral composition. Pesquisa Agropecuária Brasileira, Brasília, v. 32, n. 5, p. 533-538.

MOREIRA, A.; EVANGELISTA, A.R.; RODRIGUES. G.H.S. 1996. The alfalfa cultivars evaluation in the region of Lavras, Minas Gerais, Brazil. Pesquisa Agropecuária Brasileira, Brasília, v. 31, n. 6, p. 407-411.

MOREIRA, A.; MORAES, L.A.C.; FURLAN T.; HEINRICHS, R. 2016. Effect of glyphosate and zinc application on yield, soil fertility, yield components, and nutritional status of soybean. Communications in Soil Science and Plant Analysis, Athens v. 47, p. 1033-1047.

MOREIRA, A.; CARVALHO, J.G.; MORAES, L.A.C.; SALVADOR, J.O. 2000. Efeito de relação cálcio e magnésio do corretivo sobre micronutrientes na alfafa. Pesquisa Agropecuária Brasileira, Brasília, v. 35, n. 10, p. 2051-2056. 
NUERNBERG, N.J.; MILAN, P.A.; SILVEIRA, C.A.M. 1992. Manual de produção de alfafa. Florianópolis: EPAGRI. 102 p.

RASSINI, J.B.; FERREIRA, R.P.; MOREIRA, A.; VILELA, D. 2007. Alfalfa evaluation in region of São Carlos, São Paulo State, Brazil. Boletim de Industria Animal, Nova Odessa, v. 64, n. 4, p. 289-293, 2007.

REZENDE, P.M.; REZENDE, E.A.C.; CARVALHO P.M.E. 2007. Evaluation of soybean cultivars (Glycine max [L.] Merrill) for the Southern of Minas Gerais State. Ciência e Agrotecnologia, Lavras, v. 31, n. 6, p. 1616-1623.

SEDIYAMA, T.; PEREIRA, M.G.; SEDIYAMA, C.S.; GOMES, J.L.L. 1989. Cultura da soja: $\mathbf{1}^{\mathrm{a}}$ parte. Viçosa: Universidade Federal de Viçosa. $96 \mathrm{p}$.

VAN SOEST, P.J.; ROBERTSON, J.B.; LEWIS, B.A. 1991. Methods for dietary fiber, neutral detergent fiber, and nonstarch polysaccharides in relation to animal nutrition. Journal of Dairy Science, Riverport Lane, v. 74, n. 10, p. 3583-3597. 